\title{
Phase transitions and phase separation in a pseudospin-electron model with direct interaction between pseudospins and with transverse field
}

\author{
Yu.I.Dublenych \\ Institute for Condensed Matter Physics \\ of the National Academy of Sciences of Ukraine, \\ 1 Svientsitskii Str., 79011 Lviv, Ukraine
}

Received September 14, 2000

\begin{abstract}
The pseudospin-electron model with a direct interaction between pseudospins and with transverse field is considered. In the absence of electron transfer, the equilibrium states of the model are studied in the mean field approximation. In the $\mu=$ const regime, two types of the first order phase transitions (with jumps of the mean values of pseudospins and average electron concentration) are revealed. At $T \rightarrow 0$, phase transitions of the first type disappear, on increasing the transverse field, while those of the second type persist at any large value of the field. The phase transitions of the second type are caused by simultaneous effect of pseudospin-electron interaction and transverse field. It is also shown that in the $n=$ const regime at zero temperature, the phase separation occurs at all values of a longitudinal field $h$.
\end{abstract}

Key words: pseudospin-electron model, phase transitions, phase separation

PACS: $74.65 .+n, 71.45 . \mathrm{Gm}$

\section{Introduction}

In high temperature superconductors one can observe a series of physical phenomena which exist only separately in other types of crystals. One of the reasons for it is the strong electron correlation of the Hubbard type in the conductivity bands formed mainly by the superconducting $\mathrm{Cu}_{2}-\mathrm{O}_{2}$ planes. Another significant feature of the high- $\mathrm{T}_{\mathrm{c}}$ superconductors is the presence of strongly anharmonic elements in their structure. That can lead to various instabilities. The amplification of an effective attraction between electrons as a result of their interaction with anharmonic phonons was considered as one of the possible mechanisms of the high- $\mathrm{T}_{\mathrm{c}}$ superconductivity yet in the first publications on this subject [1-3]. 
In the best studied high- $\mathrm{T}_{\mathrm{c}}$ superconductor, $\mathrm{YBa}_{2} \mathrm{Cu}_{3} \mathrm{O}_{7-\delta}$, the strongly anharmonic elements are the so-called apical oxygen ions $\mathrm{O}_{4}$ (see e. g. [4]). To describe their vibrations, a pseudospin-electron (or Müller) model was proposed [5]. The pseudospin variable $S_{i}^{z}= \pm 1 / 2$ takes into account two possible positions of an ion $\mathrm{O}_{4}$ in a double potential well. The model includes electron-electron and pseudospin-electron interactions as well as the electron hopping. The energy of the double potential well asymmetry is considered as a longitudinal field $h$, and the energy of tunnelling splitting as a transverse field $\Omega$.

In [6] it has been shown that the electron transfer leads to the effective interaction between pseudospins. Therefore, a simpler model was proposed without electron transfer but with a direct pseudospin interaction. In [7] the phase transitions in such a model were investigated in the mean field approximation and without a transverse field. The authors showed that in the $\mu=$ const regime a first order phase transition with a jump-like behaviour of $\left\langle S^{z}\right\rangle$ is possible, and in the $n=$ const regime an instability with respect to phase separation exists in a wide range of asymmetry parameter $h$ values. A similar investigation was also performed for the two-sublattice model with direct interactions of ferroelectric type [8]. However, in the mentioned works jumps of ions $\mathrm{O}_{4}$ in double potential well were not taken into account. The aim of the present paper is to investigate the effect of the transverse field (describing the mentioned jumps) on phase transitions and phase separation in the model.

\section{Thermodynamic equilibrium conditions and thermodynamic functions in the mean field approximation}

Let us consider a pseudospin-electron system described by the Hamiltonian

$$
H=\sum_{i} H_{i}-\frac{1}{2} \sum_{i j} J_{i j} S_{i}^{z} S_{j}^{z},
$$

where

$$
H_{i}=U n_{i \downarrow} n_{i \uparrow}-\mu\left(n_{i \downarrow}+n_{i \uparrow}\right)+g\left(n_{i \downarrow}+n_{i \uparrow}\right) S_{i}^{z}-h S_{i}^{z}-\Omega S_{i}^{x}
$$

is the single-site Hamiltonian.

Here $J_{i j}$ describes the direct interaction between pseudospins $S_{i}^{z}$ and $S_{j}^{z}, U$ is the Hubbard correlation, $g$ is the pseudospin-electron interaction constant and $h$ is the longitudinal field or the energy of double potential well asymmetry. The term with the transverse field $\Omega$ is included.

In our investigation we will use the mean field approximation:

$$
S_{i}^{z} S_{j}^{z} \approx-\eta^{2}+\eta\left(S_{i}^{z}+S_{j}^{z}\right)
$$

where $\eta=\left\langle S_{i}^{z}\right\rangle$.

In this approximation the Hamiltonian has the following form:

$$
H=\sum \tilde{H}_{i}+\frac{N}{2} J \eta^{2},
$$


where

$$
\tilde{H}_{i}=U n_{i \downarrow} n_{i \uparrow}-\mu\left(n_{i \downarrow}+n_{i \uparrow}\right)+g\left(n_{i \downarrow}+n_{i \uparrow}\right) S_{i}^{z}-(h+J \eta) S_{i}^{z}-\Omega S_{i}^{x},
$$

and $J=\sum_{i} J_{i j}=\sum_{j} J_{i j}$.

The vectors $\left|n_{i \uparrow}, n_{i \downarrow}, S_{i}^{z}\right\rangle$ form a complete basic set for the Hamiltonian $\tilde{H}_{i}$ :

$$
\begin{array}{ll}
|1\rangle=|0,0,1 / 2\rangle, & |\tilde{1}\rangle=|0,0,-1 / 2\rangle, \\
|2\rangle=|1,1,1 / 2\rangle, & |\tilde{2}\rangle=|1,1,-1 / 2\rangle, \\
|3\rangle=|0,1,1 / 2\rangle, & |\tilde{3}\rangle=|0,1,-1 / 2\rangle, \\
|4\rangle=|1,0,1 / 2\rangle, & |\tilde{4}\rangle=|1,0,-1 / 2\rangle .
\end{array}
$$

In this basis the Hamiltonian $\tilde{H}_{i}$ is not diagonal, but it is easy to find a basis in which $\tilde{H}_{i}$ will be diagonal [6]. As a result we obtain the following set of eigenvalues:

$$
\begin{aligned}
& \lambda_{1, \tilde{1}}=\mp \frac{1}{2} \sqrt{\tilde{h}^{2}+\Omega^{2}}, \\
& \lambda_{2, \tilde{2}}=-2 \mu+U \mp \frac{1}{2} \sqrt{(\tilde{h}-2 g)^{2}+\Omega^{2}}, \\
& \lambda_{3, \tilde{3}}=-\mu \mp \frac{1}{2} \sqrt{(\tilde{h}-g)^{2}+\Omega^{2}}=\lambda_{4, \tilde{4}},
\end{aligned}
$$

where $\tilde{h}=h+J \eta$.

The partition function per unit cell is equal to

$$
Z_{i}=\sum_{r}\left(\mathrm{e}^{-\beta \lambda_{r}}+\mathrm{e}^{-\beta \lambda_{\tilde{r}}}\right) \mathrm{e}^{-\beta \frac{J \eta^{2}}{2}},
$$

and the total partition function is

$$
Z=Z_{i}^{N}
$$

Now we can write the Gibbs thermodynamic potential per unit cell:

$$
\omega=-\Theta \ln \sum_{r}\left(\mathrm{e}^{-\beta \lambda_{r}}+\mathrm{e}^{-\beta \lambda_{\tilde{r}}}\right)+\frac{1}{2} J \eta^{2} .
$$

From the equilibrium condition in the regime $\mu=$ const

$$
\left(\frac{\partial \omega}{\partial \eta}\right)_{T, \mu, h}=0
$$

we obtain the equation for the mean value of $\eta$ having a meaning of the order parameter:

$$
\begin{aligned}
\eta= & \frac{1}{\sum_{r}\left(\mathrm{e}^{-\beta \lambda_{r}}+\mathrm{e}^{-\beta \lambda_{\tilde{r}}}\right)}\left\{\frac{\tilde{h}}{2 \sqrt{\tilde{h}^{2}+\Omega^{2}}}\left(\mathrm{e}^{-\beta \lambda_{1}}-\mathrm{e}^{-\beta \lambda_{\tilde{1}}}\right)\right. \\
& \left.+\frac{\tilde{h}-2 g}{2 \sqrt{(\tilde{h}-2 g)^{2}+\Omega^{2}}}\left(\mathrm{e}^{-\beta \lambda_{2}}-\mathrm{e}^{-\beta \lambda_{\tilde{2}}}\right)+\frac{\tilde{h}-g}{\sqrt{(\tilde{h}-g)^{2}+\Omega^{2}}}\left(\mathrm{e}^{-\beta \lambda_{3}}-\mathrm{e}^{-\beta \lambda_{\tilde{3}}}\right)\right\} .
\end{aligned}
$$


In the case $U=2 \mu$, the curve $\eta(h)$ is symmetrical with respect to the point $(g, 0)$.

The average number of electrons per unit cell can be obtained by differentiating the expression (10) with respect to $\mu$ :

$$
n=2-2 \cdot \frac{\mathrm{e}^{-\beta \lambda_{1}}+\mathrm{e}^{-\beta \lambda_{\tilde{1}}}+\mathrm{e}^{-\beta \lambda_{3}}+\mathrm{e}^{-\beta \lambda_{\tilde{3}}}}{\sum_{r}\left(\mathrm{e}^{-\beta \lambda_{r}}+\mathrm{e}^{-\beta \lambda_{\tilde{r}}}\right)} .
$$

\section{Phase diagrams and phase separation at zero temperature}

First, let us consider the case $T \rightarrow 0$ and $\mu=$ const. Having found partially the limit we obtain the equation

$$
\begin{aligned}
\eta=\frac{1}{\mathrm{e}^{-\beta \lambda_{1}}+\mathrm{e}^{-\beta \lambda_{2}}+2 \mathrm{e}^{-\beta \lambda_{3}}}\left\{\frac{\tilde{h}}{2 \sqrt{\tilde{h}^{2}+\Omega^{2}}} \mathrm{e}^{-\beta \lambda_{1}}+\frac{\tilde{h}-2 g}{2 \sqrt{(\tilde{h}-2 g)^{2}+\Omega^{2}}} \mathrm{e}^{-\beta \lambda_{2}}\right. \\
\left.+\frac{\tilde{h}-g}{\sqrt{(\tilde{h}-g)^{2}+\Omega^{2}}} \mathrm{e}^{-\beta \lambda_{3}}\right\} .
\end{aligned}
$$

The form of this equation in the limit $T \rightarrow 0$ depends on which of the values $\lambda_{1}$, $\lambda_{2}, \lambda_{3}$ is minimal.

1. $\lambda_{1}=\min , \eta=\eta_{1}(h)=\frac{\tilde{h}}{2 \sqrt{\tilde{h}^{2}+\Omega^{2}}}$, or $\tilde{h}=\frac{2 \Omega \eta}{\sqrt{1-4 \eta^{2}}}$,

$\omega=\omega_{1}(h)=-\frac{\Omega}{2 \sqrt{1-4 \eta^{2}}}+\frac{J}{2} \eta^{2}, n=0 ;$

2. $\lambda_{2}=\min , \eta=\eta_{2}(h)=\frac{\tilde{h}-2 g}{2 \sqrt{(\tilde{h}-2 g)+\Omega^{2}}}$, or $\tilde{h}=\frac{2 \Omega \eta}{\sqrt{1-4 \eta^{2}}}+2 g$, $\omega=\omega_{2}(h)=-2 \mu+U-\frac{\Omega}{2 \sqrt{1-4 \eta^{2}}}+\frac{J}{2} \eta^{2}, n=2$;

3. $\lambda_{3}=\min , \eta=\eta_{3}(h)=\frac{\tilde{h}-g}{2 \sqrt{(\tilde{h}-g)^{2}+\Omega^{2}}}$, or $\tilde{h}=\frac{2 \Omega \eta}{\sqrt{1-4 \eta^{2}}}+g$ $\omega=\omega_{3}(h)=-\mu-\frac{\Omega}{2 \sqrt{1-4 \eta^{2}}}+\frac{J}{2} \eta^{2}, n=1$.

The curves $\eta_{i}(h)$ for cases 1,2 and 3 are parallel and their centers of symmetry are the points $(0,0),(2 g, 0),(g, 0)$, respectively. If $\Omega<\frac{J}{2}$, they have a "reverse motion", i.e., the part of a curve where the derivative $\eta^{\prime}(h)<0$. For the values of $h$ corresponding to a reverse motion the dependence $\eta_{i}(h)$ is many-valued.

The curve $\eta_{i}(h)$ as the solution of equation (14) at $T \rightarrow 0$ is composed of parts of the curves $\eta_{i}(h)$ (figure 1 ). The transition from curve 2 to curve 3 goes along the straight line

$$
h=(\mu-U) \sqrt{\frac{4 \Omega^{2}}{g^{2}-4(\mu-U)^{2}}+1}+\frac{3 g}{2}-J \eta,
$$




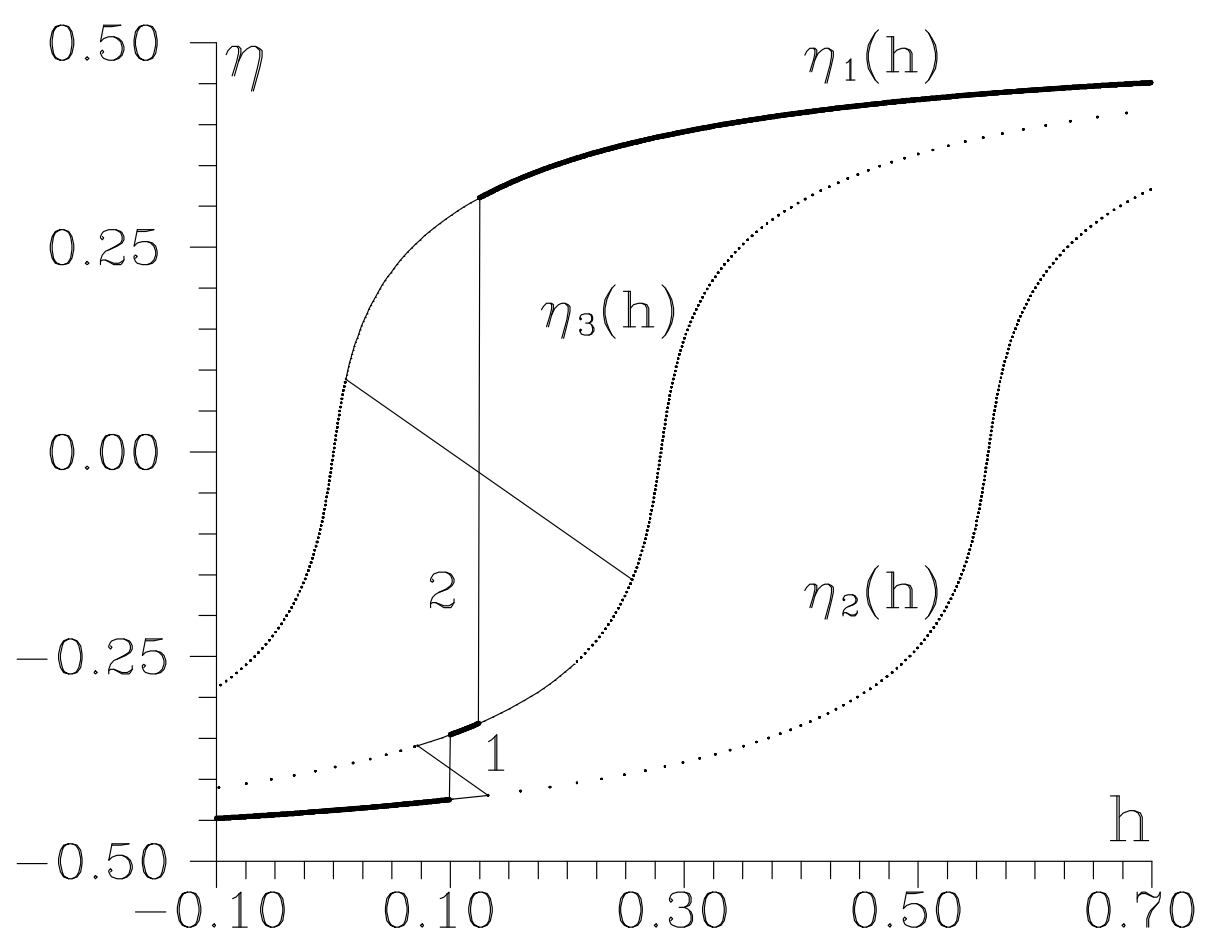

Figure 1. Order parameter $\eta$ as a function of the field $h$ (solid line) and the curves $\eta_{i}(h)$ (dotted lines). $T=0, J=1, \mu=-0.01, U=0.1, g=0.28, \Omega=0.55$. Thick lines correspond to thermodynamically stable states.

and from curve 3 to curve 1 along the straight line

$$
h=\mu \sqrt{\frac{4 \Omega^{2}}{g^{2}-4 \mu^{2}}+1}+\frac{g}{2}-J \eta .
$$

If $U>g$, the inequalities $-\frac{g}{2}+U<\mu<\frac{g}{2}+U$ and $-\frac{g}{2}<\mu<\frac{g}{2}$ are the necessary and sufficient conditions of the existence of transitions $2 \rightarrow 3$ and $3 \rightarrow 1$, respectively. However, in the case $U<g$ those inequalities are necessary but not sufficient conditions of the existence of those transitions, because if the value $\tilde{h}=$ $h+J \eta$ for the transition $3 \rightarrow 1$ is smaller than that for the transition $2 \rightarrow 3$, there is only one transition from curve 1 to curve 2 going along the straight line

$$
h=(2 \mu-U) \sqrt{\frac{\Omega^{2}}{g^{2}-(2 \mu-U)^{2}}+1}+g-J \eta .
$$

Besides the reverse motions along which transitions between the curves $\eta_{i}(h)$ occur, at the condition $\Omega<J / 2$ the resulting curve $\eta(h)$ can contain its own reverse motion of one of the curves $\eta_{i}(h)$ (figure 2 ).

The graph of the thermodynamic potential $\omega(h)$ is composed of portions of the parallel curves $\omega_{i}(h)$ connected with segments of tangent parabolas (obtained by integration of relations (15-17)). The domains where the dependence $\eta(h)$ is manyvalued correspond to self-intersections of the curve $\omega(h)$. Let $\left(h_{0}, \omega_{0}\right)$ be coordinates 
a) 0.50

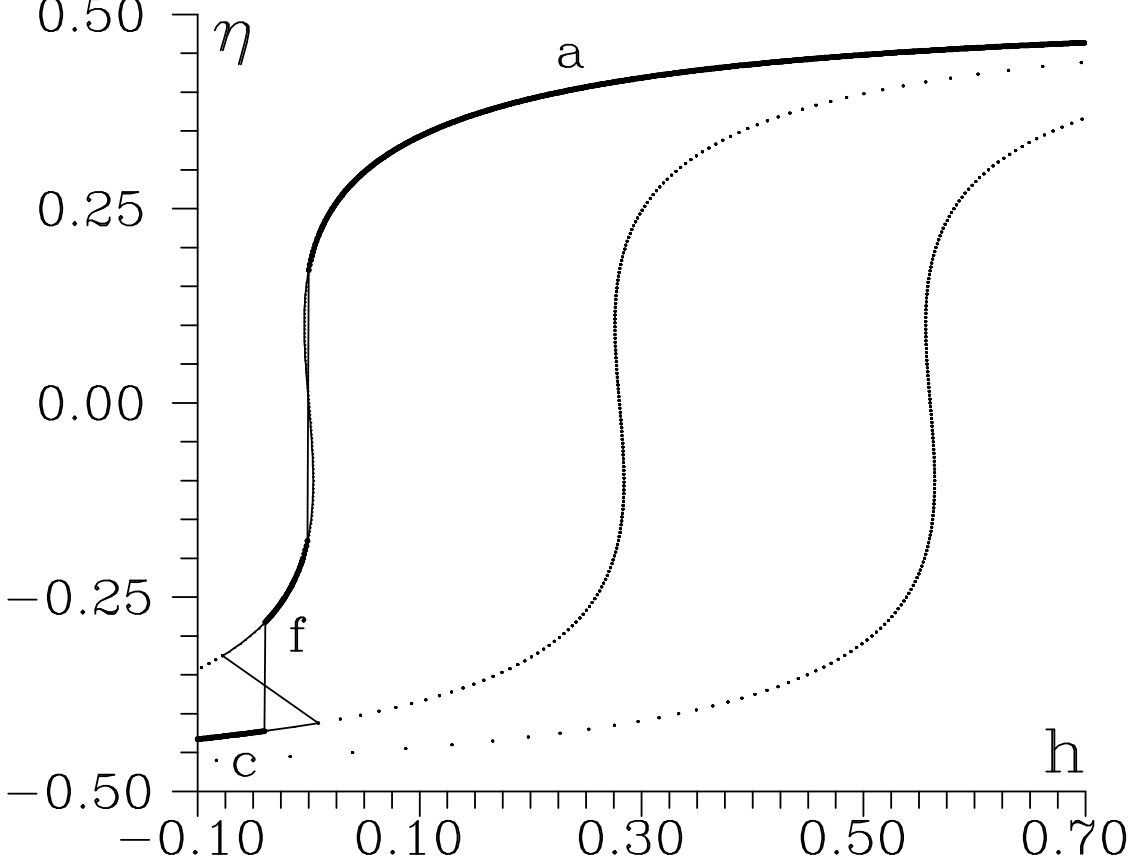

b)

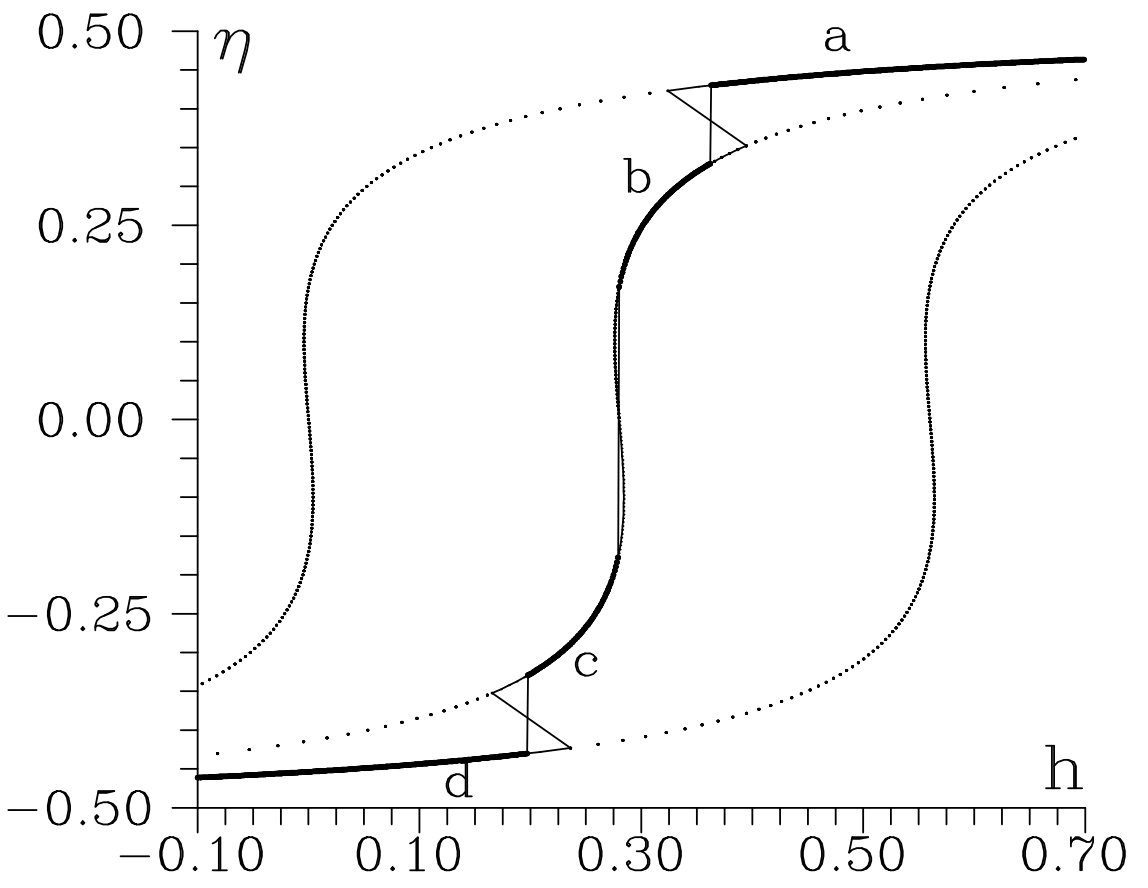

Figure 2. Cascade of phase transitions. Thick lines correspond to thermodynamically stable states, dotted lines show "not realized" parts of the curves $\eta_{i}(h)$. Letters a, b, c, d, f denote different phases. a) $T=0, J=1, \mu=-0.106$, $U=0.22, g=0.28, \Omega=0.47$; b) $T=0, J=1, \mu=0.11, U=0.22, g=0.28$, $\Omega=0.47$. 

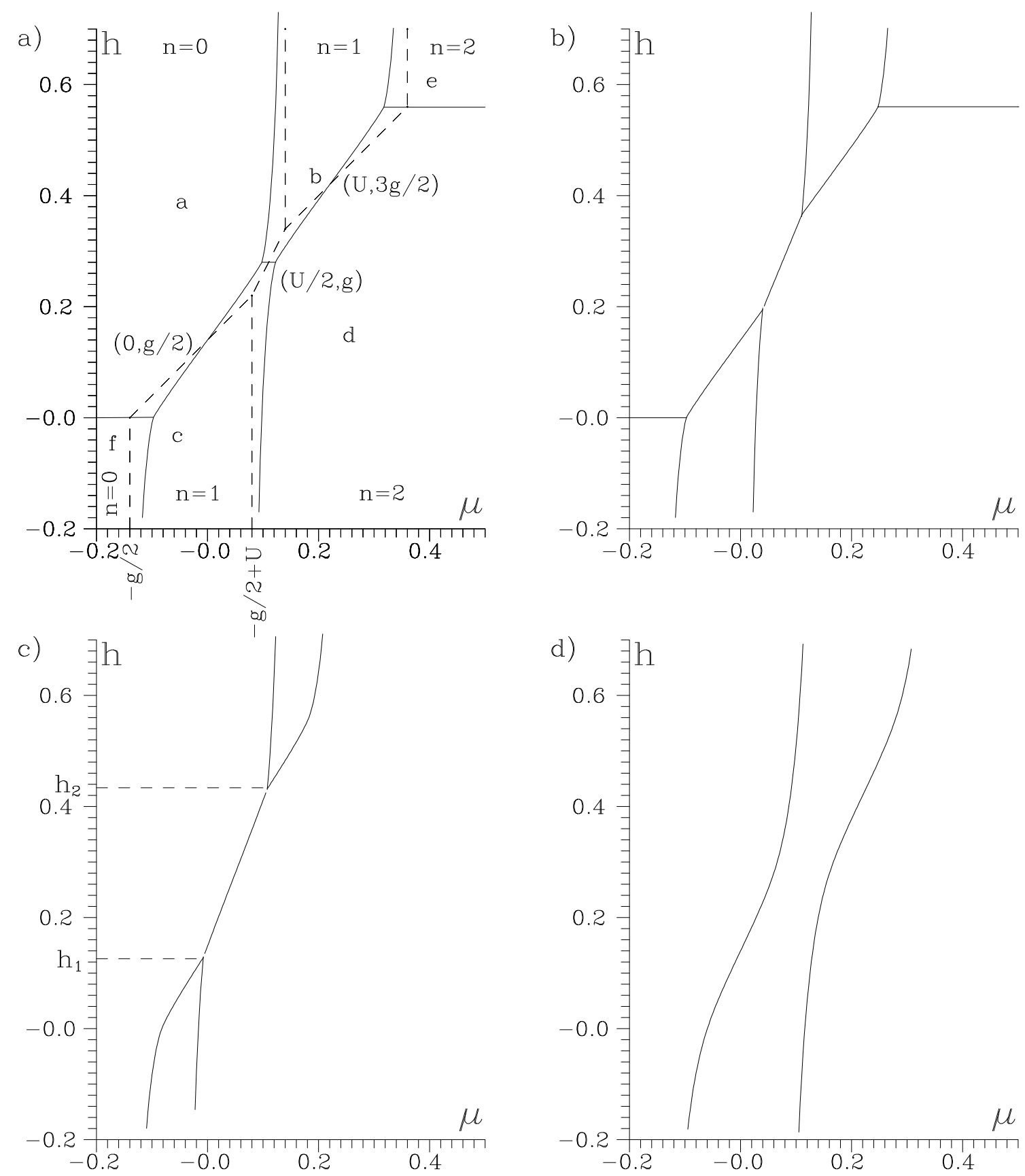

Figure 3. The $(\mu, h)$ phase diagrams at zero temperature. Dashed line shows the phase diagram at $\Omega \rightarrow 0$. Letters a, b, c, d, e, f denote different phases (see figure 2). a) $J=1, U=0.22, g=0.28, \Omega=0.47$; b) $J=1, U=0.15, g=0.28$, $\Omega=0.47$; c) $J=1, U=0.10, g=0.28, \Omega=0.55$; d) $J=1, U=0.22, g=0.28$, $\Omega=0.7$. 
of the self-intersection point. If $\omega_{0}$ is minimal of all $\omega\left(h_{0}\right)$, then at the point $h_{0}$ there is a first order phase transition, where the value of $\eta(h)$ changes by jump. The maximum possible number of reverse motions of the curve $\eta(h)$ as well as of phase transitions are three. It is significant that the phase transitions related to jumps from one curve $\eta(h)$ to another do not disappear on increasing $\Omega$ but only move along the $h$ axis. At these transitions, $\eta$ as well as $n$ has a jump. If a phase transition takes place on a single curve $\eta_{i}(h)$, then $\eta$ changes from $-\sqrt{\frac{1}{4}-\frac{\Omega^{2}}{J^{2}}}$ to $+\sqrt{\frac{1}{4}-\frac{\Omega^{2}}{J^{2}}}$ and $n$ remains constant.

The four possible topologically different diagrams in the plane $(\mu, h)$ at zero temperature are shown in figure 3. The diagrams (a) and (b) correspond to the case $\Omega<J / 2$ and the diagrams (c) and (d) correspond to the case $\Omega>J / 2$. The diagrams (b) and (c) are possible only when $U<g$. As one can see, two types of phase transitions exist at zero temperature: the first ones (horizontal lines in figure 3 (a), (b)) disappear when $\Omega$ becomes equal to $J / 2$, the other ones persist at any large value of $\Omega$. The phase transitions of the second type are caused by simultaneous effect of pseudospin-electron interaction and transverse field.

If $T \rightarrow 0$ the electron concentration $n$ can take the values 0,1 or 2 . Therefore, at fixed $n$ different from these values a phase separation occurs. In figure 4 the graph of the phase separation corresponding to the diagram of figure 3 (b) is shown. As one can see, at zero temperature the domain of phase separation encloses all values of $h$ and $n$. If $h<h_{1}$ or $h>h_{2}$, then in the case $0<n<1$ the phases with $n=0$ and with $n=1$ coexist, and in the case $1<n<2$ there are the phases with $n=1$ and with $n=2$. If $h_{1}<h<h_{2}$ the separation into phases with $n=0$ and with $n=2$ occurs. At non-zero temperature the phase separation domain is bounded.

\section{Temperature-field phase diagrams}

If temperature deviates from zero, the curve $\eta(h)$ (see figure 1) smooths out. On increasing temperature, the phase transitions disappear, first the transition 1 , then the transition 2 (figure 5).

The set of phase coexistence curves for the temperature-field phase diagram is symmetric with respect to the straight line $h=g$ if $\mu=U / 2$. The curves of the phase diagrams $\mu_{1}=U / 2-\Delta \mu$ and $\mu_{2}=U / 2+\Delta \mu$ are mutually symmetric with respect to the same straight line $h=g$, therefore it is sufficient to consider $\mu$ from the interval ] $-\infty, U / 2]$.

In the case $\Omega \geqslant J / 2$, phase transitions exist only at $-g / 2<\mu<g / 2$ or $-g / 2+$ $U<\mu<g / 2+U$.

If $\mu \rightarrow-\infty$ (or $g=0$ ) the phase coexistence line is a vertical segment of length $1 / \beta$, where $\beta=\frac{1}{\Omega} \ln \frac{J+2 \Omega}{J-2 \Omega}$. On increasing $\mu$ from $-\infty$ to $-g / 2$, this line bends more and more to the right. As soon as $\mu$ becomes greater than $-g / 2$, one more line appears in the phase diagram. On increasing $\mu$, it deforms, elongates, moves to the right along the $h$ axis, and joins the first curve (figure 6).

One can notice that the phase transitions corresponding at $T \rightarrow 0$ to jumps from 


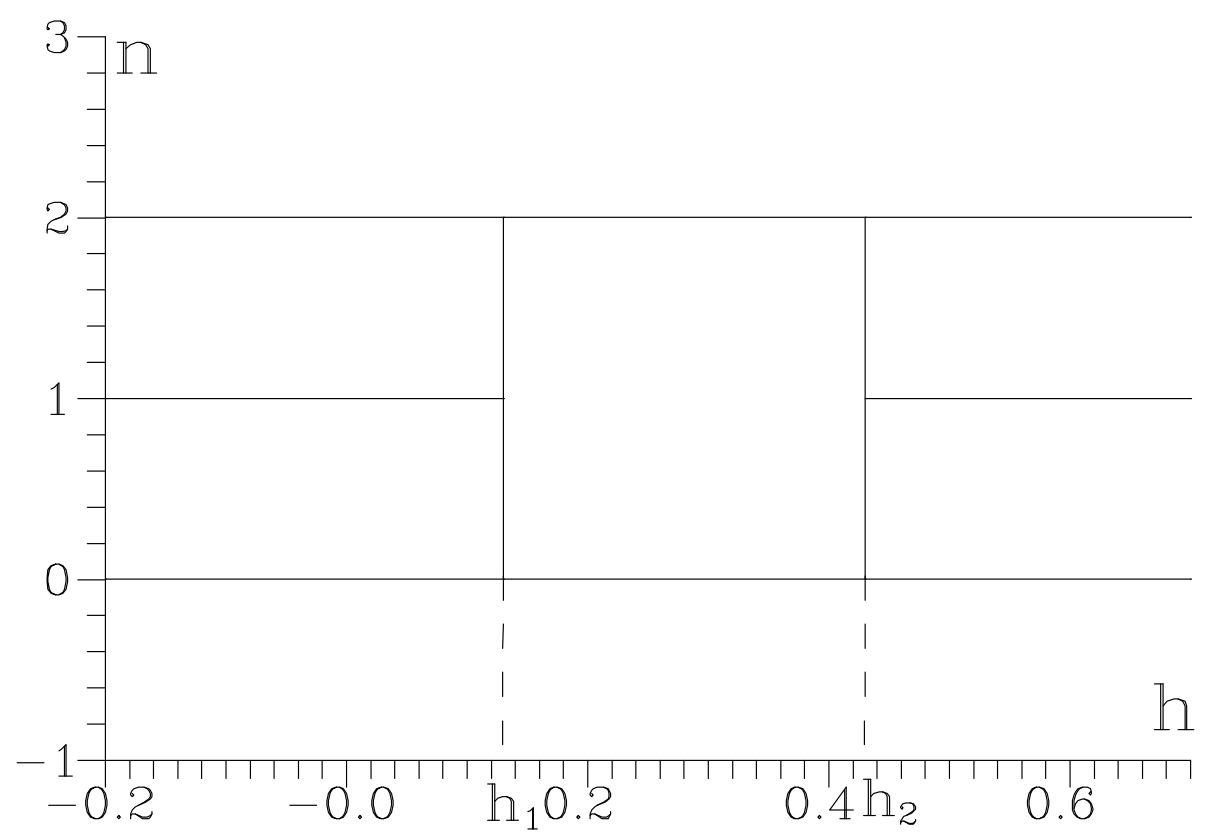

Figure 4. Phase separation at zero temperature corresponding to figure 3 (c).

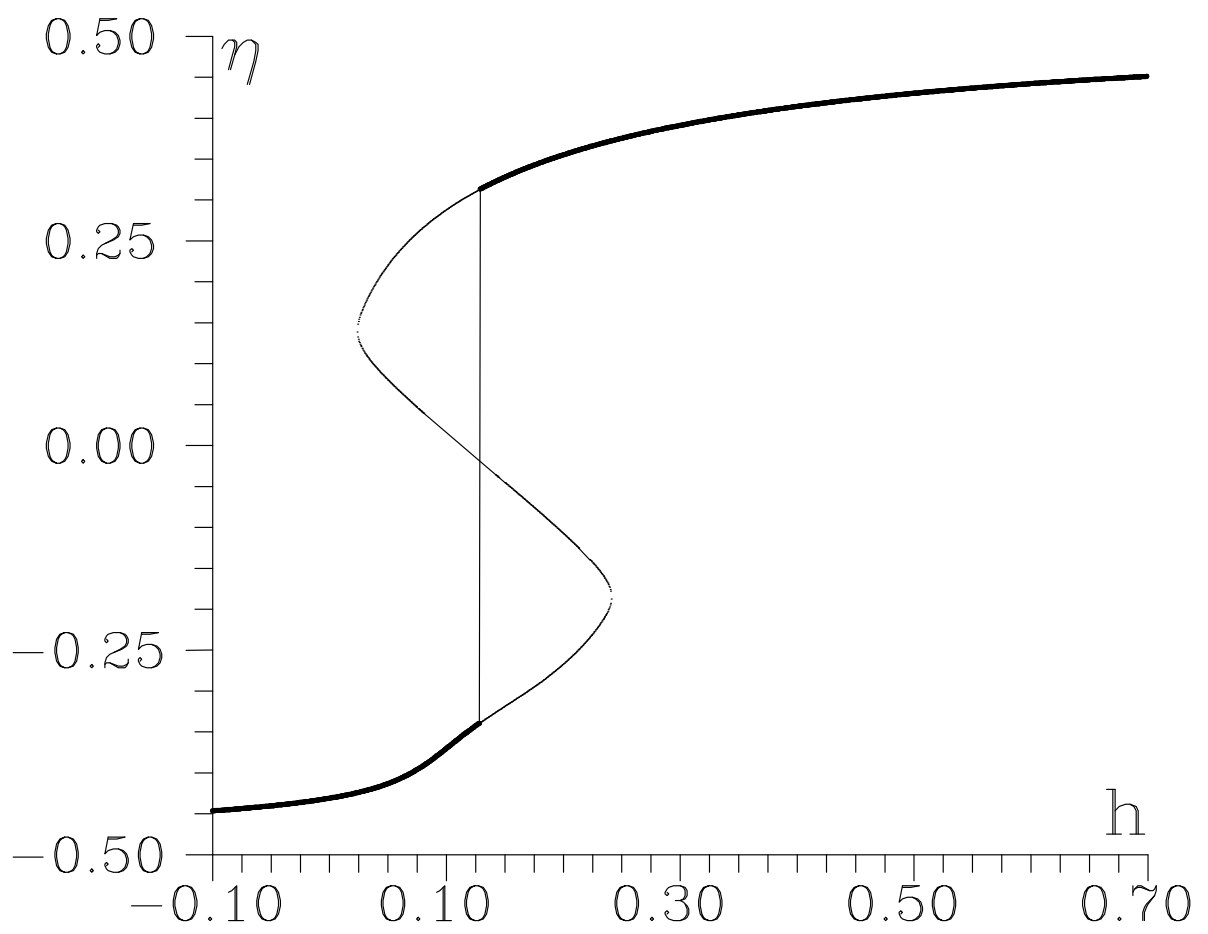

Figure 5. The order parameter $\eta$ as a function of the field $h$ at non zero temperature. $\Theta=0.003, J=1, \mu=-0.01, U=0.1, g=0.28, \Omega=0.55$. Thick lines correspond to thermodynamically stable states. 


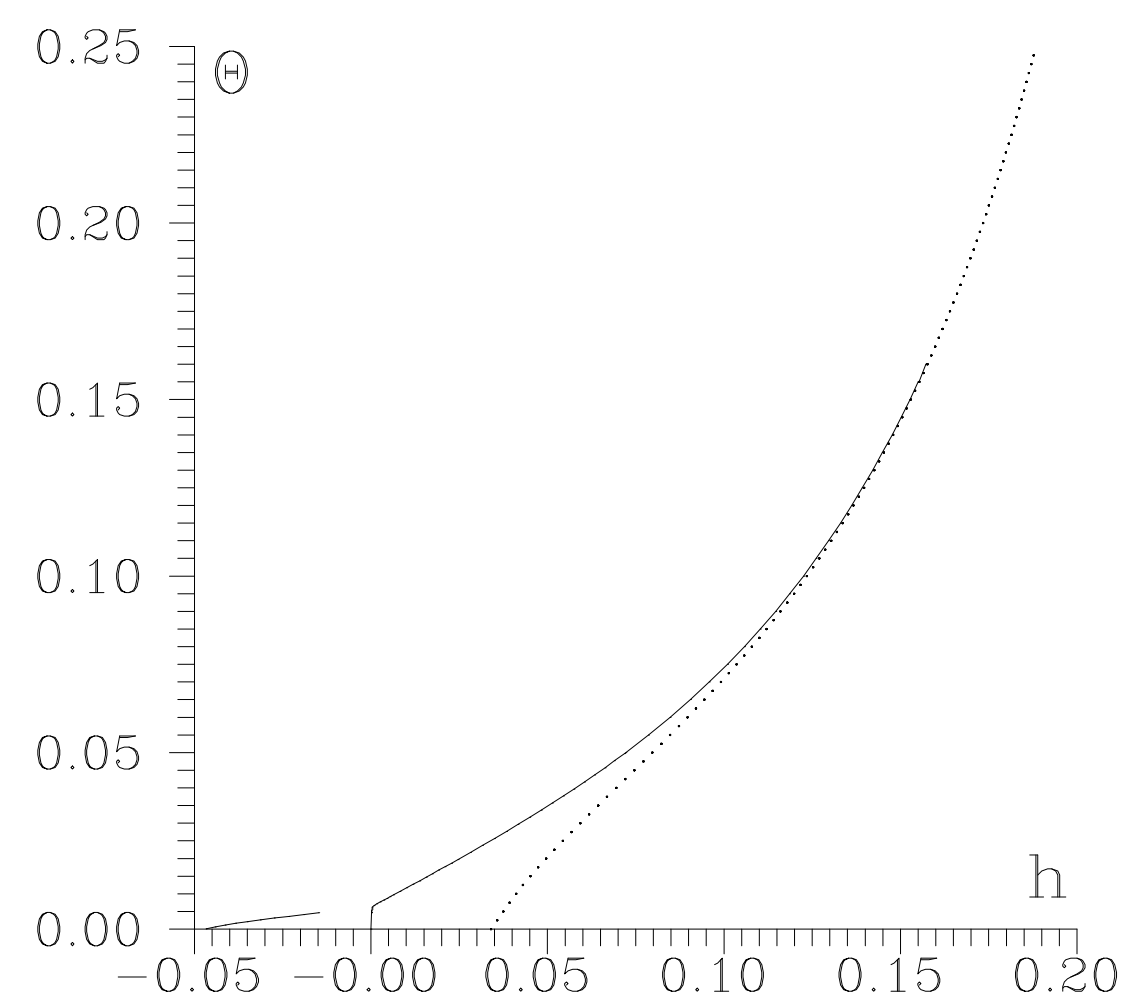

Figure 6. The $(\Theta, h)$ phase diagram. $J=1, \mu=-0.106, U=0.22, g=0.28$, $\Omega=0.47$. Dotted line shows the phase coexistence line for $\Omega=0$.

curve 2 to curve 3 or from curve 3 to curve 1 disappear well sooner on increasing temperature than those corresponding at $T \rightarrow 0$ to jumps from curve 3 to curve 1 or occurring on a single curve.

On increasing $\Omega$, the critical temperature of each transition is lowered down. As soon as $\Omega$ becomes equal to $J / 2$, transitions from curve 2 to curve 1 or those on a single curve disappear but transitions from curve 2 to curve 3 or from curve 3 to curve 1 remain at any large value of $\Omega$.

At fixed $h$ a phase transition temperature can either increase or decrease on increasing $\Omega$ (until $\Delta \eta$ becomes zero). It depends on the value of $\mu$.

\section{Results}

Hence the transverse field $\Omega$ not only reduces the critical temperature for a first order phase transition but at certain values of $\mu$ and sufficiently low temperature gives rise to one or two additional phase transitions which contrary to the case $g=0$ persist at any large value of $\Omega$.

At zero temperature, instability with respect to phase separation exists not in a bounded domain as in the case $\Omega=0$ but at any value of asymmetry parameter $h$. 


\title{
6. Acknowledgement
}

The author would like to thank professor I.V.Stasyuk for many helpful suggestions and discussions.

\section{References}

1. Kurtz S.K., Hardy J.R., Flocken J.W. // Ferroelectrics, 1988, vol. 87, p. 29.

2. Plakida N.M. // Physica Scripta, 1989, vol. T29, p. 77.

3. Bussman-Holder A., Simon A., Butner H. // Phys. Rev. B, 1989, vol. 39, p. 207.

4. Müller V., Hucho C., Maurer D. // Ferroelectrics, 1992, vol. 130, p. 45-76.

5. Müller K.A. // Z. Phys. B., 1990, vol. 80, p. 193; Phase Transitions, 1998 (Special issue).

6. Stasyuk I.V., Shvaika A.M. // Acta Physica Polonica A, 1993, vol. 84, p. 293.

7. Stasyuk I.V., Havrylyuk Yu. // Cond. Matt. Phys., 1999, vol. 2, p. 487.

8. Stasyuk I.V., Dublenych Yu.I. Phase transitions and phase separation in a pseudospinelectron model with direct interaction of antiferroelectric type. Preprint of the Institute for Condensed Matter Physics, ICMP-99-07U, Lviv, 1999, 26 p.

\section{Фазові переходи та розділення фаз у псевдоспін-електронній моделі з прямою взаємодією псевдоспінів та поперечним полем}

\author{
Ю.І.Дубленич \\ Інститут фізики конденсованих систем НАН України, \\ 79011 Львів, вул. Свєнціцького, 1 \\ Отримано 14 вересня 2000 р.
}

В наближенні середнього поля досліджено рівноважні стани псевдоспін-електронної моделі з прямою взаємодією псевдоспінів та з поперечним полем за відсутности перенесення електронів. У режимі $\mu=$ const виявлено фазові переходи першого роду (зі стрибкоподібною зміною середнього значення псевдоспіна та середньої електронної концентрації) двох типів: перші у випадку $T \rightarrow 0$ зникають зі збільшенням поперечного поля, інші залишаються, яким би великим не було це поле. Фазові переходи другого типу зумовлені одночасним впливом псевдоспін-електронної взаємодії та поперечного поля. Показано також, що в режимі $n=$ const за нульової температури відбувається розшарування фаз в усій області значень поздовжнього поля $h$.

Ключові слова: псевдоспін-електронна модель, фазові переходи, розділення фаз

PACS: $74.65 .+n, 71.45 . \mathrm{Gm}$ 
